\title{
The impact of dietary saturated fat replacement with unsaturated fat on the plasma lipidome and cardiometabolic disease risk
}

\author{
L. Sellem ${ }^{1}$, F. Eichelmann ${ }^{2}$, M. Weech ${ }^{1}$, K.G. Jackson ${ }^{1}$, M. Schulze ${ }^{2}$ and J.A. Lovegrove ${ }^{1}$ \\ ${ }^{1}$ Hugh Sinclair Unit of Human Nutrition, University of Reading, Whiteknights, Reading, UK and \\ ${ }^{2}$ Department of Molecular Epidemiology, German Institute of Human Nutrition Potsdam-Rehbruecke, Nuthetal, \\ Germany
}

Evidence from epidemiological studies and randomised controlled trials (RCTs) suggests that replacing dietary saturated (SFAs) with unsaturated fatty acids (UFA) may have beneficial impacts on cardiometabolic disease (CMD) risk ${ }^{(1)}$. However, interdisciplinary research narrowing the gap between interventional and observational evidence is lacking. Recent findings have suggested the utility of high-throughput lipidomics to identify potential CMD risk markers and provide novel aetiological insights into the relationship between dietary fat composition and $\mathrm{CMD}$ risk $^{(2)}$. Thus, this study aimed to assess the lipidome-mediated impact of replacing dietary SFAs with UFAs on CMD risk.

Plasma fatty acid (FA) concentrations among 14 lipid classes were measured using high-throughput lipidomics analyses (Metabolon, USA) in samples from the DIVAS parallel RCT $(\mathrm{n}=113)$, which investigated the effects of three 16-week diets enriched in SFAs (target SFA:monounsaturated fatty acids MUFA: $n-6$ polyunsaturated fatty acids PUFA ratio $=17: 11: 4 \%$ total energy TE), MUFAs (target SFA:MUFA: $n$ - 6 PUFA ratio $=9: 19: 4 \%$ TE), or a mixture of UFAs (target SFA:MUFA: $n-6$ PUFA ratio $=9: 13: 10 \%$ TE) on CMD risk markers such as fasting lipid profiles, and markers of inflammation, endothelial function, and arterial stiffness ${ }^{(3)}$. Similar lipidomics analyses were conducted on samples from two case-cohorts from the EPIC-Potsdam prospective cohort study [ $\mathrm{n}=$ $1,707$ for type 2 diabetes (T2D) and $n=1,886$ for cardiovascular diseases (CVD) $]^{(2)}$. Within-class FAs sensitive to the DIVAS dietary intervention were identified using multiple linear regression models and related to CMD risks in each EPIC-Potsdam case-cohort using multivariable Cox proportional hazard models. Finally, within- class FAs associated with changes in CMD risk markers assessed in the DIVAS study were identified using constraint-based feature selection algorithms and multiple linear regression models.

Analysis of within-class plasma FA concentrations revealed high-UFA intervention diets from the DIVAS study broadly reduced the concentrations of FAs associated with higher CVD risk, and to a lesser extent T2D risk, in the EPIC-Potsdam cohort, such as palmitic (16:0) and stearic (18:0) acids in di- and triacylglycerol, and myristic acid (14:0) in hexosylceramides, with clearer effects of the high- MUFA diet compared to the mixed-UFA. Reciprocally, the high-UFA diets increased the concentrations of FAs associated with lower CMD risk, such as erucic acid (22:1) in triacylglycerol and nervonic acid (24:1) in lactosylceramides. Furthermore, increased low-density lipoprotein cholesterol and total cholesterol concentrations were associated with a higher abundance of arachidic acid (20:0) in cholesteryl esters and diacylglycerol $\left(p<10^{-3}\right.$ and $p=0.001$, respectively), whilst increased interleukin- 6 and P-selectin concentrations were associated with higher proportions of arachidic acid $(20: 0)$ in mono- $(p=0.008)$ and triacylglycerol $(\mathrm{p}=0.02)$.

Overall, these findings suggest a potential mediating role of plasma lipid metabolites in the association between dietary fat and CMD risk. Future research combining interventional and observational findings and investigating the identified within-class FAs is warranted to improve our understanding of dietary fat composition in CMD aetiology.

\section{References}

1. Scientific Advisory Committee on Nutrition: Saturated fats and health (2019).

2. Eichelmann F, Sellem L, Wittenbecher C et al. (2021), under review (Circulation).

3. Vafeiadou K, Weech M, Altowaijri H et al. (2015) Am J Clin Nutr 102(1), 40-48. 